\title{
Insuficiencia renal crónica: cuando se pierde la autonomía
}

\author{
Chronic renal failure: when the autonomy is lost \\ Insuficiência renal crônica: quando o intervalo é perdido \\ Raquel Alba Martín \\ DUE Especialista en Salud Mental. USM Hospital Reina Sofía (Córdoba)
}

Cómo citar este artículo en edición digital: Alba Martín, R. (2015). Insuficiencia renal crónica: cuando se pierde la autonomía. Cultura de los Cuidados (Edición digital), 19, 42. Disponible en: http://dx.doi.org/10.14198/cuid.2015.42.04>

Correspondencia: Calle Hermano Bonifacio, 3. 14520-Fernán Núñez (Córdoba)
Correo electrónico: raquelalbamartin@satse.es
Recibido: 12/01//2015; Aceptado: 10/06/2015

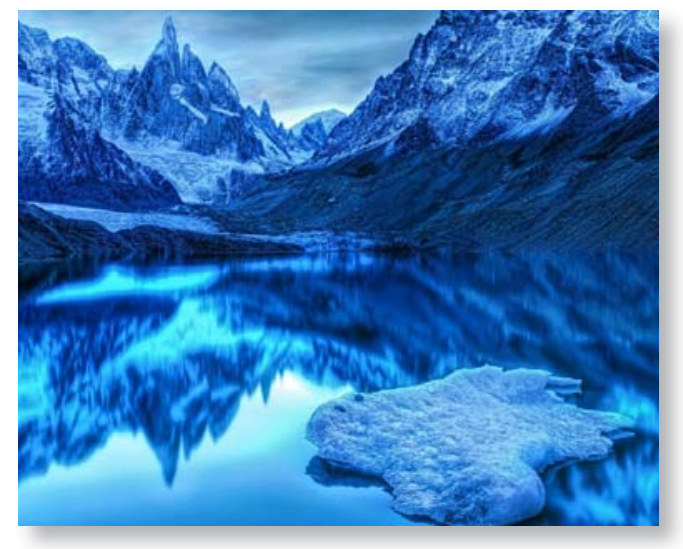

\section{ABSTRACT}

Introduction: The IRC is one of the most common causes of healthcare related not only to the patient's physical health but also psychological level.

Objective: to explore the experiences and feelings experienced by a patient with CKD.

Methodology: includes a single participant using convenience sampling. Data by patient history and an in depth interview with open questions were collected for 45 minutes. Consultation took place in nursing; after informing the confidentiality of your personal data, and the possibility of abandonment at any time. The conversation was recorded and transcribed in full by the same person, performing notes on the observations noted in a field notebook. Af- ter reading the transcript of the data showed the reporting compliance, and performing the verification thereof. For analysis of the content of the analytical sequence Taylor-Bogdan adapted continued. Findings: Through repeated readings of the text a list of themes, ideas, concepts and propositions that allowed to define the categories of study was drawn.

Conclusions: The discourse of our patient shows the memory of their disease at 64 , debuting with big changes in their life and great concern and anxiety about the future situation.

Keywords: nephrological nursing; perception of disease; chronic renal failure; integral health care; patient safety.

\section{RESUMO}

Introdução: O IRC é uma das causas mais comuns de saúde relacionados, não só para a saúde física do paciente, mas também a nível psicossocial.

O objetivo deste estudo é explorar as experiências e sentimentos vivenciados por um paciente com doença renal crônica.

Metodologia: Inclui um único participante por amostra de conveniência. Foram coletados dados de histórico do paciente e uma entrevista em profundidade com perguntas abertas para 45 minutos. Realizou-se na consulta de enfer- 
magem; depois de informar a confidencialidade dos seus dados pessoais, bem como a possibilidade de abandono a qualquer momento. A conversa foi gravada e transcrita na íntegra pela mesma pessoa, fazendo anotações em observações se destacar em um caderno de campo. Depois de ler a transcrição dos dados mostrou a relatórios de conformidade, também realizada a verificação dos mesmos. Para a análise do conteúdo da sequência analítica Taylor-Bogdan adaptado continuou. Resultados: Por repetidas leituras do texto uma lista de tópicos, idéias, conceitos e proposições que permitiram definir as categorias de estudo foi desenhado.

Conclusões: $\mathrm{O}$ discurso do nosso paciente mostra a memória de sua doença em 64, que estréia com grandes mudanças em sua vida e de grande preocupação e ansiedade sobre a situação futura.

Palavras-chave: enfermagem em nefrologia. percepção de doença. irc. atenção integral à saúde. segurança do paciente.

\section{RESUMEN}

Introducción: La IRC es una de las causas más frecuentes de atención sanitaria relacionada no sólo con la salud física del paciente sino también a nivel psicosocial.

El objetivo del presente estudio es explorar las vivencias y sentimientos que experimenta un paciente con Insuficiencia Renal Crónica.

Metodología: Se incluye a un solo participante mediante muestreo por conveniencia. Se recolectaron los datos mediante la historia clínica del paciente y una entrevista en profundidad con preguntas abiertas durante 45 minutos. Tuvo lugar en la consulta de enfermería; tras informarle de la confidencialidad de sus datos personales, y de la posibilidad de abandono en cualquier momento. La conversación fue grabada íntegramente y transcrita por la misma persona, realizándose apuntes sobre las observaciones a destacar en un cuaderno de campo. Después de leer la transcripción de los datos la informante mostró su conformidad, realizándose así la verificación de los mismos. Para el análisis del contenido se siguió la secuencia analítica adaptada de Taylor-Bodgan. Resultados: Mediante lecturas repetidas del texto se confeccionó una lista con los temas, ideas, conceptos y proposiciones que permitieron definir las categorías de estudio.

Conclusiones: El discurso de nuestro paciente muestra el recuerdo de la evolución de su enfermedad a sus 64 años, que debuta con grandes cambios en su situación vital y gran preocupación por el futuro e inquietud.

Palabras clave: enfermería nefrológica. percepción de enfermedad. irc. atención integral de salud. seguridad del paciente.

\section{INTRODUCCIÓN}

El bienestar psicológico, las vivencias y pensamientos de pacientes con enfermedad crónica es lo que ha motivado mi interés por realizar este estudio, a alguien con una interesante historia para ser leída, desde el punto de vista de la profesional que escribe.

Estoy hablando de un gran educador que vio mermada su carrera profesional por una Insuficiencia Renal Crónica (Miguelez, Rodríguez, Ruiz, Cardera, Huertas y Morales, 1991). Una persona con un estilo de vida saludable e inigualable se enfrenta a una década de sufrimiento por una enfermedad hereditaria que “da la cara” a sus 49 años.

Todo empezó tras sufrir una lipotimia en sus clases de educación física, además de sintomatología inespecífica y de difícil diagnóstico tal como ruborización, aumento de tensión ar- 
terial puntual, caídas tras los desmayos y fatiga frecuente.

Atemorizado y absorto anta la incertidumbre de no saber qué le ocurría, los servicios de urgencias deciden derivarlo al hospital para la realización de las pruebas diagnósticas correspondientes, sin suerte de detectar motivo alguno de enfermedad.

Llegado el otoño, su malestar se acrecienta y se decide su ingreso en un hospital privado para una segunda opinión médica. Tras meses interminables, según relata nuestro informante, y múltiples pruebas complementarias alteradas sin diagnóstico certero, el cirujano responsable decide realizarle una nefrectomía parcial (extirpación de riñón) por sospecha de tumoración maligna principalmente en el riñón derecho.

Dura intervención quirúrgica cuando los demorados resultados de anatomía patológica muestran la benignidad de las tumoraciones días después. Pero ya estaba hecho. El riñón extraído y su organismo incapaz de funcionar normalmente con uno solo...

Este relato motivó mi necesidad de profundizar en el tema en cuestión y tras una revisión bibliográfica exhaustiva de vivencias de enfermedad renal crónica, decidí embarcarme en conocer cómo se sienten y/o afrontan la enfermedad aquellas personas que obligatoriamente tienen que prejubilarse por incapacidad total, cuando disfrutan vocacionalmente de su trabajo. Me impresionó escuchar su agradecimiento a los profesionales que lo han atendido, a pesar del error médico que condicionó su insuficiencia renal, cuando su padecimiento era una enfermedad poliquística. El hecho de afrontarla positivamente y esa perseverancia por mantenerse con vida.

Por todo ello y después de años de lucha en diálisis, visité a nuestro protagonista y le pedí, previo consentimiento, que me escribiera en un diario sus recuerdos y vivencias, con todo detalle posible, desde el inicio de la enfermedad.

Posteriormente, sería leído por él para su grabación, análisis del contenido y transcripción integral. (Amezcua y Hueso, 2004; Taylor y Bogdan, 2008). El resultado de dicha narración, con la limitación de su temblor residual en las manos, queridos lectores, es el del "Rol de un buen paciente" y se muestra a continuación fragmentada en categorías temáticas.

\section{BIBLIOGRAFÍA}

- Amezcua M. y Hueso C. (2004). Cómo elaborar un relato biográfico. Arch Memoria (Revista digital) Disponible en: http://www.indexf.com/memoria/metodologia.php [Última consulta el 26 de enero de 2015]

- Miguelez, M.C., Rodríguez, L., Ruiz, P., Cardera, M., Huertas, M.C., Morales y O.I.. (1991). Estudio de ansiedad y depresión en pacientes urológicos. Asociación Española de ATS en Urología. Congreso XIII. 1990, 40:69-70, Burgos.

- Porter, M. (2000). Manual para Excelencia en la Investigación mediante Grupos Focales. Academia para el Desarrollo Educativo. Washington D.C: Healthcom.

- Taylor, S.J. y Bogdan R. (2008). Introducción a los métodos cualitativos de investigación. Barcelona: Ed. Paidós Ibérica.

- Vela, A. (2009). Fundamentos y metodología de la Investigación Cualitativa. Guía Académica, III Edición, Arequipa; Disponible en: http://www.monografias.com/ trabajos87/fundamentos-y-metodologia-investigacioncualitativa/fundamentos-y-metodologia-investigacioncualitativa2.shtml [Última consulta el 26 de enero de 2015]

\section{RELATO BIOGRÁFICO}

\section{LA ENFERMEDAD- INTERVENCIÓN}

Al despertar, sientes que el funcionamiento del cuerpo es normal. No hay nada extraño salvo dolores, herida, etc. Posteriormente, in- 
tentas comprobar cómo está la bolsa de orina. Satisfacción grande al observar líquido en la misma: El riñón está funcionando. [Sonríe]. Cuando se está enfermo, se siente la necesidad de otras personas. Hay una dependencia a gran escala de esas personas [...].

\section{PENSAMIENTOS Y VIVENCIAS}

A partir de ahí, tus pensamientos se dirigen a conseguir realizar lo que los profesionales te ordenan. Tu vida va encaminada a protegerte: La medicación a su hora, vida tranquila, revisiones, alimentación y ejercicio. [Suspira].

Comparas tu vida con la anterior y notas la gran diferencia del estado físico de tu cuerpo. Hasta llegas a sentirte mucho mejor que antes. Poco a poco consigues olvidar el transplante que no llega, se hace rutinaria la medicación. Sólo vuelves a preocuparte cuando llega la revisión. Si el médico te dice: "todo está bien", vuelves a perder el miedo que llevas. De esta manera notas que tu ánimo paralelamente se siente más optimista.

\section{LOS PROFESIONALES}

Los profesionales que buscan la forma de atenderte de la mejor manera, de hacerte pasajera y agradable esa enfermedad. Y más, cuando esa enfermedad es crónica y necesitas diariamente de sus servicios.

\section{LA FAMILIA}

Los familiares que siempre encuentran la forma de hacerte la vida más placentera y llevadera. Te animan, te prestan sus tiempos con abnegación, vigilan tus estados de ánimo y velan los momentos físicos más dolorosos [...].

\section{SU JUBILACIÓN Y EL DESEADO TRAS-} PLANTE

He leído que esta semana ha habido en el Hospital Reina Sofía bastantes trasplantes por varias donaciones. Me llamaron el martes durante la diálisis y ví el trasplante más cerca. Le das a la cabeza informaciones constantes de tu estado de ánimo y de cómo será la vida después de ese trasplante. Piensas el cambio tan grande que experimentará tu vida, sobre todo la dependencia que hay de otras personas y el sacrificio que llevan por atenderte. Buscas la manera de organizar la nueva etapa que se presenta en tu vida y el modo de agradecer el bien que está recibiendo tu cuerpo por la solidaridad de otras personas.

\section{REFLEXIONES FINALES}

Por todo ello, siempre se debe agradecer el esfuerzo y la práctica que cumplen todos ellos en el discurrir de tu enfermedad.

Hay que comprender sus pequeños fallos en la tarea diaria, pues son más por el celo que ponen en ello que por fallo en sí. Muchas veces eso ocurre por cansancio, por querer acumular tareas que deben ser hechas más espaciosamente.

Felicitemos el trabajo y dedicación que estas personas realizan por la pronta recuperación de la enfermedad del paciente y adquiera una forma más optimista en su devenir, convirtiéndola en una "enfermedad saludable".

Me gustaría terminar mi relato con una cita del Concilio Vaticano II que siempre tengo presente: "La dignidad humana requiere que el hombre actúe siembre según su conciencia y libre elección. Los cristianos deben tener conciencia de ello y combatirlo con máxima energía”. 